\title{
Suicidio y crítica cultural en la medicina española del siglo XIX
}

\author{
José Javier Plumed Domingo (*) y Enric J. Novella $(* *)$ \\ $\left({ }^{*}\right) \quad$ orcid.org/0000-0002-7467-3414. Hospital Universitario La Fe (Valencia). Universidad de Valencia. \\ jplumed@terra.com \\ ${ }^{* *}$ ) orcid.org/0000-0001-6823-563X. Área de Historia de la Ciencia. Universidad Miguel Hernández. \\ enovella@umh.es
}

Dynamis

[0211-9536] 2015; 35 (1): 57-81

http://dx.doi.org/10.4321/S0211-95362015000100003
Fecha de recepción: 3 de febrero de 2014

Fecha de aceptación: 15 de octubre de 2014

SUMARIO: 1.- Introducción. 2.-EI desorden de las pasiones. 3.-Suicidio y cambio social. 4.--Suicidio, degeneración y fin de siglo. 5.-Conclusiones.

RESUMEN: El presente trabajo explora el destacado papel del suicidio en la crítica cultural desplegada por los médicos españoles del siglo XIX a través de un análisis de los principales modelos teóricos que inspiraron sus aportaciones en torno a la causación del mismo. En la primera mitad del siglo, el factor etiológico más discutido fueron las pasiones, las cuales, de acuerdo con el espiritualismo dominante, actuaban en tensión permanente con un yo reflexivo, consciente y dueño de su libre albedrío. Posteriormente, y en el contexto de una progresiva somatización de los fenómenos morales e intelectuales, la concepción del suicidio como un acto libre del individuo fue modificándose hasta considerarlo como una consecuencia más o menos directa de ciertas alteraciones orgánicas. Pero este proceso no anuló el lugar central de las conductas suicidas en el marco de la crítica cultural decimonónica, pues, con la introducción de la teoría de la degeneración, los médicos dispusieron de una doctrina que les permitía conciliar el determinismo biológico con la muy extendida percepción de una crisis moral y social que amenazaba la estabilidad y los logros de la sociedad burguesa.

PALABRAS CLAVE: suicidio, etiología, crítica cultural, medicina, España, siglo XIX.

KEY WORDS: suicide, etiology, cultural criticism, medicine, Spain, 19th century. 


\section{Introducción}

Como es sabido, el suicidio constituye un fenómeno cuyas coordenadas sociales y culturales han despertado siempre un gran interés historiográfico ${ }^{1}$. Desde el punto de vista de la historia de la ciencia, uno de los aspectos más interesantes y estudiados ha sido el proceso de secularización y medicalización de las conductas suicidas operado en el tránsito a la modernidad y consumado en gran medida a lo largo del siglo XIX ${ }^{2}$; no en vano, el suicidio y su relación más menos directa con la enfermedad mental fueron uno de los ámbitos de reflexión y elaboración conceptual más insistentemente cultivados por la medicina decimonónica.

En este sentido, una cuestión que ha dominado la atención de los historiadores ha sido la comprobación empírica del (supuesto) aumento en la frecuencia de las conductas suicidas observado por los médicos del Ochocientos y, en suma, del nexo causal entre civilización moderna y suicidio reiteradamente postulado por muchos de ellos. Así, por ejemplo, y en contra de las apreciaciones sugeridas en trabajos anteriores sobre el llamado «efecto Werther» ${ }^{3}$, Jeffrey Watt ha afirmado que, teniendo en cuenta los datos procedentes de los archivos de la ciudad de Ginebra, sí hubo una epidemia de suicidios en el tránsito del siglo XVIII al XIX. En su opinión, la causa fundamental de este fenómeno fue el mismo desarrollo de la sociedad burguesa, que implicó un enriquecimiento de las clases medias y altas en relación con las bajas, de manera que, ante las nuevas expectativas de riqueza de un determinado sector de la población,

1. Una buena revisión bibliográfica se ofrece en Healy, Róisín. Suicide in early modern and modern Europe. The Historical Journal. 2006; 49 (3): 903-919.

2. Véanse, a modo de ejemplo: MacDonald, Michael. The medicalization of suicide in England: Laymen, physicians, and cultural change, 1500-1870. The Milbank Quarterly. 1989; 67 (S1): 69-91; McDonald, Michael; Murphy, Terence R. Sleepless souls. Suicide in early modern Europe. Oxford: Oxford University Press; 1990; Watt, Jeffrey, ed. From sin to insanity. Suicide in early modern Europe. Ithaca and London: Cornell University Press; 2004; Martínez Pérez, José. La construcción de una nueva identidad social: alienismo y suicidio en la Francia del siglo XIX. In: Fernández Sastre, José Luis; Fuentenebro, Filiberto; Rojo, Águeda, eds. Suicidio. Madrid: Sociedad de Historia y Filosofía de la Psiquiatría; 2008, p. 39-72; Plumed Domingo, José Javier; Rojo Moreno, Luis. La medicalización del suicidio en la España del siglo XIX: Aspectos teóricos, profesionales y culturales. Asclepio. 2012; 64 (1): 147-166.

3. Steinberg, Holger. Der Werther-Effekt. Historischer Ursprung und Hintergrund eines Phänomens. Psychiatrische Praxis. 1999; 26: 37-42. 
aumentó la frustración de quienes no podían alcanzarla ${ }^{4}$. Sin embargo, y dada la escasez de datos epidemiológicos fiables, la mayoría de los autores han aducido diversos factores profesionales e ideológicos para explicar el uso constante de la bandera del suicidio como una señal del peligro de la modernidad. Pues, en líneas generales, no cabe duda de que el recurso continuado de los médicos a la conducta suicida como un (claro) indicador de malestar social y un (acuciante) problema de salud pública no buscaba sino reafirmar su nuevo papel como gestores y garantes de la integridad del «hombre intelectual y moral» ${ }^{5}$.

En su conocida historia del suicidio en la cultura occidental, George Minois ha señalado cómo el primer tercio del siglo XIX asistió a una verdadera eclosión de explicaciones morales del mismo que subrayaban los riesgos del nuevo espíritu materialista vinculado al progreso económico, así como el efecto disolvente de los ideales de libertad y autonomía alentados por las revoluciones burguesas ${ }^{6}$. De este modo, si unas décadas antes los sabios ilustrados - con la notable excepción de Kant- habían clamado por la despenalización del suicidio e incluso lo habían reivindicado como un acto legítimo de soberanía individual - es el caso de Montesquieu, Voltaire o Hume-, el periodo posrevolucionario lo convirtió en el objeto de una intensa preocupación compartida por un importante sector de la profesión médica ${ }^{7}$. Un artículo aparecido en 1836 en los Annales d'hygiène publique et de médecine légale, la publicación francesa más importante en su campo, sintetiza a la perfección el sentir de muchos médicos europeos del momento:

«El daño que el suicidio hace a la sociedad no consiste en absoluto en el número de hombres que se lleva, sino en el desaliento contagioso que propaga;

\footnotetext{
4. Watt, Jeffrey. Choosing death. Suicide and calvinism in early modern Geneva. Kirksville: Truman State University Press; 2004.

5. Véanse, en este sentido, Martínez Pérez, José. Conductas autodestructivas y salud de un Estado: sobre el suicidio como indicador del bienestar social a través de su empleo por la psiquiatría francesa (1800-1856). In: Montiel, Luis, ed. La salud en el Estado de Bienestar. Madrid: Editorial Complutense; 1993, p. 79-106; Martínez Pérez, José. Suicidio, crisis política y medicina mental en la Francia del siglo XIX (1801-1885). Frenia. 2001, I (1): 39-65; y Novella, Enric J. Medicina, antropología y orden moral en la España del siglo XIX. Hispania. 2010; 70 (236): 709-736.

6. Minois, George. History of suicide. Voluntary death in Western culture. Baltimore: The Johns Hopkins University Press; 1999, p. 319.

7. McManners, John. Death and the Enlightenment. Changing attitudes to death among Christians and unbelievers in eighteenth-century France. Oxford: Clarendon Press; 1981, p. 436-437; Lieberman, Lisa. Leaving you. The cultural meaning of suicide. Chicago: Ivan Dee; 2003, p. 13-37.
} 
en la incredulidad por todo, en el Dios de su religión, en el hombre, en la sociedad [...]. El suicidio, [...] como un tifus contagioso, debilita las fuerzas y el coraje de quienes lo respiran en el aire. Para ellos, las contrariedades se transforman en desgracias y las decepciones pasajeras en calamidades desesperantes, y estos cadáveres [...] no hacen más que infectar el lugar donde yacen. El suicidio reviste pues una importancia más grande de lo que se cree en su primera apariencia: es un verdadero mal cuya progresión es preciso detener instantáneamente» ${ }^{8}$.

Desde el punto de vista de la naciente medicina mental, Howard I. Kushner ha mostrado cómo la mayoría de sus pioneros adoptaron, al menos hasta la década de 1870, un planteamiento etiológico ambiental y moralista centrado en el potencial patógeno de unas pasiones aberrantemente estimuladas por las tensiones sociales, culturales y políticas resultantes del nuevo espíritu de los tiempos ${ }^{9}$. De hecho, y tal como apunta Zilla Gabrielle Cahn, el propio Philippe Pinel ya había relacionado el suicidio con los efectos de la Revolución y las pasiones incontroladas de las masas, a las que hacía responsables de un buen número de cuadros melancólicos con «una inclinación irresistible al suicidio» ${ }^{10}$. Y, en una línea muy similar, su discípulo Jean-Étienne-Dominique Esquirol - que no lo consideraba como una enfermedad en sí misma, sino como un «síntoma consecutivo dependiente casi siempre del delirio de las pasiones o de algún tipo de alienación mental» ${ }^{11}$ no dudaba en señalar que:

8. Citado por Martínez Pérez, n. 5, 2001, p. 57.

9. Kushner, Howard I. American psychiatry and the cause of suicide, 1844-1917. Bulletin of the History of Medicine. 1986; 60 (1): 36-57. Sobre las pasiones como el factor etiológico fundamental y hasta cierto punto constitutivo del primer alienismo pueden pueden consultarse Gauchet, Marcel y Swain, Gladys. La pratique de l'esprit humain. L'institution asilaire et la révolution démocratique. Paris: Gallimard; 1980; Pigeaud, Jackie. Le rôle des passions dans la pensée médicale de Pinel à Moreau de Tours. History and Philosophy of the Life Sciences. 1980; 2: 123-140. Weiner, Dora. Mind and body in the clinic: Philippe Pinel, Alexander Crichton, Dominique Esquirol and the birth of psychiatry. In: Rousseau, George, ed. The languages of the psyche. Mind and body in Enlightenment thought. Berkeley: California Press; 1990; p. 331-401; y Huneman, Philippe. Montpellier vitalism and the emergence of alienism in France (1750-1800): the case of the passions. Science in Context. 2008; 21: 615-647.

10. Cahn, Zilla Gabrielle. Suicide in French thought from Montesquieu to Cioran. Peter Lang: New York; 1998, p. 175-185.

11. Esquirol, [Jean-Étienne-Dominique]. Suicide. In: Dictionnaire des Sciences Médicales. Paris: C.L.F. Panckoucke; 1821, Vol. LIII, p. 213-283 (275). 
«cuando más desarrollada se encuentra la civilización, cuando más se excita el cerebro y aumenta la susceptibilidad, cuando más se incrementan las necesidades y los deseos son más imperiosos, cuando más se multiplican las causas de los pesares, y cuando más abunda la alienación mental, más suicidios debe haber» ${ }^{12}$.

En cierto modo, los esfuerzos iniciales de los alienistas por elaborar un modelo médico convincente de las conductas suicidas mediante conceptos y categorías clínicas como la «melancolía suicida» o la «monomanía suicida» contrastaban con su tendencia a atribuir su causación a los cambios sociales de la época, hasta el punto de que algunos de ellos llegaron a cuestionar que muchos suicidios pudieran relacionarse directamente con la enfermedad mental. Así, por ejemplo, el francés Alexandre Brierre de Boismont afirmaba en 1850 que:

«la enfermedad del tedio vital, incluso con tendencias suicidas, no puede considerarse como una variedad de la locura, a menos que vaya acompañada de un trastorno de los sentimientos y de las facultades intelectuales. Querer convertir una enfermedad moral en un apéndice de la locura es agotar una rica fuente de observaciones, y justifica el reproche que tan a menudo se dirige a los alienistas de ver sus asuntos en todas partes» ${ }^{13}$.

Pero, en líneas generales, el recurso a las pasiones como el factor etiológico común de la locura y las conductas autodestructivas permitió soslayar durante décadas cualquier contradicción que amenazara la comprensión del suicidio como un fenómeno esencialmente (psico)patológico, pues, no por casualidad, si había más suicidios en Europa era en gran medida porque - como apuntaba el mismo Brierre de Boismont- también «la alienación es más frecuente y sus formas más diversas» ${ }^{14}$.

12. Esquirol, n. 11, p. 248. Sobre la posición de Esquirol con respecto al suicidio, que ha sido objeto de apreciaciones divergentes, puede verse Berrios, Germán E. The history of mental symptoms. Descritive psychopathology in the nineteenth century. Cambridge: Cambridge University Press; 1996, p. 443-454.

13. Brierre de Boismont, Alexandre. De l'ennui (Tædium vitae). Annales Médico-Psychologiques. 1850; 2 (2S): 545-585, p. 561. Este ensayo tuvo pocos años después una traducción castellana: Brierre de Boismont, Alexandre. Del fastidio de la vida. Boletín del Instituto Médico Valenciano. 1854; 5: 158-161, 171-177, 189-193, 201-204, 217-222, 233-236.

14. Brierre de Boismont, Alexandre. De l'influence de la civilisation sur le développement de la folie. Annales d'Hygiène Publique et de Médecine Légale. 1839; 21: 241-295. 
En cualquier caso, es sabido que en la segunda mitad del siglo XIX los planteamientos etiológicos dominantes de la medicina mental se desplazaron definitivamente desde las pasiones hacia las lesiones y alteraciones anatómicas y fisiopatológicas ${ }^{15}$. Así, las conductas suicidas empezaron a ser vistas desde una óptica mucho más determinista en la que la predisposición hereditaria fue cobrando un protagonismo cada vez mayor ${ }^{16}$. Y este proceso llegó a su apogeo con el degeneracionismo, para el que la sociedad era, en última instancia, una fuerza controlada (y amenazada) por los mecanismos responsables de la degradación biológica de la especie humana ${ }^{17}$. En consecuencia, el suicidio aparecía ahora como uno de los síntomas y enfermedades sociales resultantes del proceso degenerativo; pero este punto de vista no hizo sino reforzar el potencial de las conductas suicidas como un instrumento de crítica cultural, pues, con el degeneracionismo, los médicos, y muy especialmente los psiquiatras, dispusieron finalmente de una doctrina que les permitía conciliar el determinismo biológico con la muy extendida percepción de una crisis moral y social que ponía en riesgo la estabilidad y los logros de la sociedad burguesa ${ }^{18}$.

15. Sobre las múltiples razones de este giro teórico y discursivo pueden verse Lantéri-Laura, Georges La chronicité dans la psychiatrie française moderne, Annales ESC. 1972; 27: 548568; Clark, Michael J. The rejection of psychological approaches to mental disorder in late nineteenth-century British psychiatry. In: Scull, Andrew, ed. Madhouses, mad-doctors and madmen. The history of psychiatry in the Victorian era. Philadelphia: University of Pennsylvania Press; 1981, p. 241-270; Jacyna, LS. Somatic theories of mind and the interests of medicine in Britain (1850-1879). Medical History. 1982; 26: 233-258; y Álvarez, Raquel; Huertas, Rafael; Peset, José Luis. Enfermedad mental y sociedad en la Europa de la segunda mitad del siglo XIX. Asclepio. 1993; 45 (2): 41-60.

16. Véase, a modo de ejemplo, Saury, Honoré. Étude clinique sur la folie héréditaire (les dégénérés). París: Adrien Delahaye et Émile Lecrosnier; 1886, p. 8.

17. Sobre el degeneracionismo y su vertiente psiquiátrica pueden consultarse Pick Daniel. Faces of degeneration. A European disorder (1848-1918). Cambridge: Cambridge University Press; 1989; y Huertas, Rafael. Locura y degeneración. Psiquiatría y sociedad en el positivismo francés. Madrid: CSIC; 1987.

18. La influencia de la degeneración y el evolucionismo sobre la concepción del suicidio fue muy importante en muchos países europeos. Así, Susan Morrisey ha mostrado cómo el degeneracionismo inspiró todos los tratados sobre el suicidio redactados en Rusia a partir de 1890. Morrisey, Susan. Suicide and the body politic in Imperial Russia. Cambridge: Cambridge University Press; 2006, p. 190-194. A lo largo del trabajo hacemos uso del concepto «crítica cultural» en un sentido amplio, esto es, dentro de la muy influyente tradición de reflexión crítica en torno a la problematicidad de la cultura y la civilización insistentemente cultivada por la intelectualidad europea desde finales del siglo XVIII. Véase, por ejemplo, Bollenbeck, Georg. Eine Geschichte der Kulturkritik. Von Rousseau bis Günther Anders. Munich: C.H. Beck; 2007. 
Teniendo en cuenta este marco general, el presente trabajo examina las particularidades del tratamiento del suicidio en la medicina española del siglo XIX a través de un análisis detenido de las principales reflexiones y aportaciones en torno a las causas del mismo. Como veremos, el suicidio tuvo una presencia destacada en la producción de los médicos españoles, cuyas especulaciones etiológicas reprodujeron en gran medida los grandes modelos teóricos del alienismo francés, aunque les imprimieron un sesgo conservador derivado de la persistente influencia del catolicismo y la amplia proyección del espiritualismo psicológico en el país. Posteriormente, la irrupción del positivismo y el organicismo psiquiátrico atenuó por un tiempo la condena moral y el uso ideológico del suicidio, pero la circulación de las ideas degeneracionistas volvió a situarlo entre las más preocupantes enfermedades sociales del fin de siglo español.

\section{El desorden de las pasiones}

En las décadas centrales del siglo XIX, uno de los autores más influyentes en cuanto a la comprensión del suicidio en España fue el francés Jean-Baptiste Félix Descuret, cuyo libro La medicina de las pasiones (1841) fue prontamente traducido al castellano por el higienista catalán Pedro Felipe Monlau y se convirtió en una referencia constante para los autores de orientación más conservadora $^{19}$. En su obra, Descuret presentaba un modelo de individuo ajustado al dogma católico en el que el yo, identificado con el alma, era debilitado por la acción permanente de unas pasiones estrechamente asociadas a las ideas de tentación y pecado. No obstante, el individuo contaba con una autonomía suficiente que le permitía decidir libremente sobre sus actos a pesar de las limitaciones a las que se veía sometido. El suicidio era, por tanto, un acto voluntario - aunque esta voluntad estuviese menoscabada por efecto del estímulo pasional-y, en consecuencia, no era sino un «triple atentado contra la moral, contra Dios y contra sí mismo» ${ }^{20}$. De este modo,

19. Descuret, Jean Baptiste-Félix. La medicina de las pasiones o las pasiones consideradas con respecto a las enfermedades, a las leyes y a la religión. Barcelona: Imprenta de Antonio Bergnes y Cia. 1842. Sobre la recepción de Descuret en España pueden consultarse Diéguez, Antonio. Perspectivas sobre las pasiones en la España del período romántico. Frenia. 2010; 10: 29-48; y Novella, Enric J. La medicina de las pasiones en la España del siglo XIX. Dynamis. 2011; 31 (2): 453-473.

20. Descuret, n. 19, p. 364. 
Descuret no proponía un modelo estrictamente médico del suicidio, pues, aunque concedía la posibilidad de que en ocasiones fuera un síntoma de verdadera alienación, creía firmemente que, en última instancia, el sujeto estaba en posesión de su libertad y sus actos mantenían (casi siempre) una dimensión moral.

Otro autor de gran influencia entre los médicos españoles fue el ya citado Brierre de Boismont ${ }^{21}$, que en 1856 reunió sus investigaciones sobre el suicidio en un volumen que se convirtió de inmediato en una importante obra de referencia ${ }^{22}$. En un ensayo anterior sobre el «tedio vital» cuya versión castellana fue publicada por el Boletín del Instituto Médico Valenciano, Brierre ya había expuesto detalladamente su visión sobre las causas del fenómeno ${ }^{23}$. Así, establecía dos tipos: aquellos suicidios producidos como consecuencia directa de los delirios y alucinaciones de un sujeto enfermo, y aquellos en los que el individuo no sufría un trastorno en la percepción de la realidad. En estos últimos casos, el suicidio se producía como consecuencia de la presión excesiva que las pasiones ejercían sobre el yo, un mecanismo patogénico que también conducía a los individuos al crimen o a cualquier mala acción ${ }^{24}$. En su opinión, el freno social y educacional que tradicionalmente se había impuesto a las pasiones había dejado de actuar adecuadamente en los tiempos modernos, y ello había favorecido un claro aumento en la prevalencia de las conductas suicidas.

La medicina española, profundamente condicionada hasta los años 60 del siglo XIX por el catolicismo, identificaba la voluntad con el concepto de alma, con lo que la psicología se ajustaba al dogma católico. Recordemos que hasta la aparición de la Ley de Libertad de Enseñanza del 21 de Octubre de 1868 no desapareció la censura oficial de la Iglesia Católica sobre los libros que pudieran cuestionar el dogma. A partir de este momento hubo una actitud más abierta hacia las ideas que pudieran cuestionar la religión imperante que se frenó a partir de 1875, ya que, con la restauración monárquica, la sociedad española estuvo fuertemente polarizada entre conservadurismo

21. Sobre la influencia de este autor en la psiquiatría española puede verse Novella, Enric J.; Huertas, Rafael. Alexandre Brierre de Boismont and the origins of the Spanish psychiatric profession. History of Psychiatry. 2011; 22: 387-402.

22. Brierre de Boismont, Alexandre. Du suicide et de la folie suicide. Paris: Baillière; 1856. Véase si no la entusiasta reseña que le dispensó el American Journal of Insanity (1856; 12: 352).

23. Brierre de Boismont, n. 13, 1854.

24. Brierre de Boismont, n. 22, p. 352. 
católico y liberalismo y hubo un agrio debate entre estas dos tendencias ${ }^{25}$. En consecuencia, y frente a los planteamientos materialistas que pretendían negar el libre albedrío, el suicidio se interpretó repetidamente como un ejemplo del fracaso de la voluntad del individuo, que con ello caía en el más grave pecado. En este sentido, el médico Miguel Hernández Montero señalaba que el suicidio era justamente un fenómeno que invalidaba las tesis materialistas. Según este autor, las pasiones eran los «resortes de los que la naturaleza se vale para mover la voluntad», por lo que necesitaban un «principio inteligente» que las orientase y guiase, «el alma racional» ${ }^{26}$. Y si ésta se encontraba moralmente afectada, las pasiones influían libre y perniciosamente sobre el individuo.

Esta cuestión se debatió en una serie de sesiones celebradas en 1849 en el Instituto Médico Valenciano, donde el médico catalán Francisco Castellví y Pallarés presentó una memoria ${ }^{27}$ que fue finalmente publicada junto con su réplica, redactada por Francisco Trullet y Antonio Coca ${ }^{28}$. En este texto, muy influido por Descuret, Castellví definía al sujeto de acuerdo con la doctrina espiritualista como un «principio de unidad inmaterial, espiritual, dotado de facultades propias que no son del organismo» y caracterizado por la «conciencia del yo», esto es, influido por lo físico aunque solo dependiente de sí mismo para pensar ${ }^{29}$. En su opinión, las pasiones debilitaban tanto al yo - que, por lo común, las manejaba voluntariamente condicionado por el ambiente ideológico y moral de la sociedad- que podían conducirlo hasta la locura. Y, en consecuencia, el suicidio debía ser considerado como una enfermedad, ya que era prácticamente imposible

25. Pelayo, Francisco. Ciencia y creencia en España durante el siglo XIX. Madrid: CSIC; 1999, p. 135 y 342.

26. Hernández Montero, Miguel. Consideraciones fisiológicas sobre las funciones mentales y afectivas. Gaceta Médica. 1849; 5: 125-127, 141-143, 149-152, 157-159, 173-175 (157).

27. Castellví y Pallarés, Francisco, Memoria sobre el suicidio dedicada al muy ilustre Instituto Valenciano. Boletín del Instituto Médico Valenciano. 1849; 2: 466-471, 473-475, 482-485, 493499, 513-521. Sobre este autor pueden consultarse: Rey González, Antonio (1984), Clásicos de la psiquiatría española del siglo XIX (V): Francisco Castellví y Pallarés (1812-1879), Revista de la Asociación Española de Neuropsiquiatría, 1984, 4, 103-116; y Novella, Enric J. El discurso del yo: El espiritualismo psicológico en la cultura española de mediados del siglo XIX. Asclepio. 2013; 65 (2): 16.

28. Trullet, Francisco; Coca, Antonio. Dictamen que da la Comisión de Medicina y Cirugía acerca de la memoria sobre el suicidio dedicada al Instituto Médico Valenciano por su socio D. Francisco Castellví. Boletín del Instituto Médico Valenciano. 1849; 2: 485-487, 489-493, 502-505.

29. Castellví y Pallarés, n. 27, p. 518. 
que la reflexión y la voluntad, elementos definitorios de la cordura, no se encontraran dañados en el suicida.

La respuesta de Trullet y Coca en nombre de la Comisión de Medicina y Cirugía de la corporación valenciana matizó los punto de vista de Castellví y Pallarés, subrayando la gradualidad de la influencia de las pasiones en la producción del suicidio; en un primer momento, las pasiones podían inducir ideas suicidas, pero todavía permitían que el individuo ejerciese su libertad, con lo que éste cometía un acto pecaminoso si consumaba el acto suicida. «Deben admitirse - decía la comisión- entre el perfecto juicio y el estado de demencia, grados intermedios caracterizados por el dominio no absoluto de la pasión» ${ }^{30}$. De este modo, Trullet y Coca evitaban el riesgo ideológico y moral que todavía implicaba en ese momento una asunción más plena de la medicalización del suicidio.

En líneas generales, pues, cabe señalar que los autores de orientación más conservadora consideraron las pasiones como una fuerza psíquica del sujeto y no como una mera tensión física. Al ser dirimidas por el yo - que era el encargado de contenerlas en un precario equilibrio- y no tratarse de un balance de tensiones biológicas regido por las leyes de la fisiología, las pasiones constituían un vehículo adecuado para la noción católica de tentación moral, con lo que podían definirse peyorativamente de acuerdo con los dictados de la moralidad vigente. Así, citando a Descuret, un doctorando de la Universidad Central señalaba en 1864 que las pasiones no estaban relacionadas ni con el alma ni con el cuerpo, sino que eran «independientes de la materia a la que resisten» ${ }^{31}$. Por su parte, el médico madrileño Joaquín Quintana y Ollero, autor de una importante memoria sobre las pasiones presentada a finales de 1862 en la Real Academia de Medicina de Madrid, también negaba que fueran una función meramente física, aunque estuvieran condicionadas por el organismo y su medio. En este sentido, la monomanía, esto es, la categoría clínica en la que tendían a encuadrarse entonces las conductas suicidas, no era para él sino una consecuencia de la presión de las pasiones sobre una conciencia libre ${ }^{32}$. Y, de la misma forma, uno de los autores más destacados de las filas espiritualistas,

\footnotetext{
30. Trullet, Francisco; Coca, Antonio, n. 28, p. 503.

31. Moreno y López, Venancio. ¿Cuáles son los caracteres diferenciales de la monomanía y la pasión? Toledo: Imprenta de López Fando; 1864, p. 26.

32. Quintana, Joaquín. Pasión y locura: distinción fundamental entre ambos estados. El Siglo Médico. 1863; 10: 212-215, 227-230, 244-247, 261-265 (227).
} 
el palentino Matías Nieto y Serrano, subrayaba que las funciones pasionales y mentales no eran dependientes de las orgánicas, sino que presentaban un desarrollo paralelo que no interfería con éstas últimas ${ }^{33}$.

Más proclives a asumir un modelo organicista de las pasiones, a mediados de la centuria un número creciente de médicos españoles pretendieron despojar definitivamente al suicidio de su carácter moralmente oprobioso insistiendo en el carácter irresistible de las fuerzas internas que impulsaban al individuo a poner fin a su vida. Siguiendo sobre todo a Esquirol, estos autores pensaban que las pasiones eran fenómenos biológicos capaces de obrar una serie de cambios físicos en el cerebro y los órganos internos. Así, por ejemplo, Raimundo Monasterio y Correa, traductor al español del célebre Tratado completo de las enajenaciones mentales publicado originalmente por el alienista francés en 1838, definía las pasiones como fuerzas «no dominables», afirmando la existencia en el ser humano de un instinto destructivo que, aunque pudiera ser modulado por la educación, podía degenerar en una fuerza incontrolable:

«En la organización del hombre hay varios agentes, varias fuerzas de impulsión o movimiento más fuertes que su voluntad. Y siendo esto así, ¿qué razón tendríais para negar que el infeliz que haya perdido esa potencia cometa un crimen, un asesinato, por más que repugne a su conciencia, a la moral y a la legislación?» ${ }^{34}$.

Por su parte, el futuro pionero de la escuela histológica española Aureliano Maestre de San Juan también planteó en su tesis doctoral sobre el suicidio que las pasiones eran capaces de causar cambios físicos muy acusados en el organismo. De hecho, su acción continuada producía una

33. Nieto y Serrano, Matías. Dictamen de la Sección de Filosofía sobre la memoria de Don Joaquín Quintana titulada Pasión y Locura. El Siglo Médico. 1863; 10: 275-278. Este modelo teórico no se mostraba muy distante del defendido en su momento por Philippe Pinel. Así, por un lado, Pinel pensaba que las pasiones estaban estrechamente ligadas a un sustrato somático, pero, por el otro, también las explicaba desde una perspectiva psicológica y las consideraba modificables y susceptibles de cambio por el tratamiento moral. Como ejemplo, proponía la utilidad de someter al suicida a una fuerte impresión que modificase su estado pasional para hacerle así desistir de su intento. Desde este punto de vista, pues, la locura no asentaba en una lesión orgánica identificable anatomopatológicamente, sino que era un trastorno funcional y reversible. Véase Pinel, Philippe. Tratado médico-filosófico de la enagenación del alma o manía. Madrid: Imprenta Real; 1804, p. 329-332.

34. Monasterio y Correa, Raimundo. ¿Existe la monomanía homicida? Madrid: Imprenta de Santa Coloma y Peña; 1851, p. 18. 
lesión que llevaba al sujeto a perder su capacidad de decidir, hasta el punto de caer en un estado de delirio agudo que podía conducirle al suicidio. En consecuencia, describía el llamado "suicidio crónico» de acuerdo con el modelo de Esquirol, esto es, como el resultado de la actividad prolongada de una pasión deprimente que provocaba cambios estables en el sistema nervioso ${ }^{35}$.

En cualquier caso, el autor que defendió una concepción de las pasiones más cercana al organicismo fue sin duda el incansable Pedro Mata y Fontanet, catedrático de medicina legal en la Universidad Central y figura de enlace entre las corrientes sensualistas de origen dieciochesco y el nuevo positivismo que empezaba a dominar el pensamiento médico europeo ${ }^{36}$. En su libro sobre la libertad moral, Mata rechazaba el cargo de materialismo del que le acusaban reiteradamente los autores espiritualistas y describía la voluntad como un equilibrio de fuerzas condicionado por factores somáticos ${ }^{37}$. En relación al suicidio, en su Tratado de medicina y cirugía legal refería casos de individuos cuya voluntad se veía impotente para frenar una tendencia congénita a esta conducta: «para cada sujeto que se mata con serenidad, calma y previsión, gozando de razón cabal — sentenciabahay infinitos que la tienen perdida ${ }^{38}$. En su opinión, pues, el suicidio era la consecuencia de una pasión violenta que normalmente actuaba sobre un individuo predispuesto; y esta predisposición era el resultado de un variopinto conjunto de factores constitucionales, entre los que mencionaba el temperamento sanguíneo, la herencia, el perfil instintivo o la mayor o menor sensibilidad a verse influido por factores externos como el clima.

35. Maestre de San Juan, Aureliano. ¿Qué causas conducen al hombre a poner fin a su vida? Madrid: Imprenta del Colegio de Sordomudos y Ciegos; 1851, p.6. Sobre esta obra puede verse Doménech, Edelmira. Un aspecte poc conegut de l'obra d'Aureliano Maestre de San Juan: la seva tesi sobre el suicidi (1851). Gimbernat. 1990; 13: 65-80.

36. Sobre las ideas psicológicas de Mata pueden verse Domenech, Edelmira. Las ideas psicológicas de Pedro Mata en el campo de la psicología de su tiempo. Asclepio. 1980; 32: 137-150; y López Fernández, M. a Nieves. La psicología en la obra de Pedro Mata y Fontanet. Valladolid: Universidad de Valladolid; 1993. Y sobre las interminables polémicas que libró con los representantes más destacados de la escuela espiritualista, puede verse nuevamente Novella, n. 27.

37. Mata y Fontanet, Pedro. De la libertad moral o libre albedrío. Madrid: Bailly-Baillière; 1868, p. 130-131.

38. Mata y Fontanet, Pedro. Tratado de medicina y cirugía legal, 5. a ed. Madrid: Bailly-Baillière; 1875, Vol. III, p. 482. 


\section{Suicidio y cambio social}

Ya hemos visto que desde principios del siglo XIX se venía haciendo un gran hincapié en el peso que los cambios sociales tenían sobre las causas del suicidio. De hecho, se ha afirmado que una de las causas por las que las pasiones cobraron entonces una importancia clave en la etiología de la enfermedad mental fueron los cambios revolucionarios y las tensiones sociopolíticas generadas en el tránsito del siglo XVIII al XIX, periodo que conllevó una transformación dramática en la estructura y la organización social proveniente del Antiguo Régimen ${ }^{39}$.

Asimismo, también hemos visto que el modelo vigente en el segundo tercio del siglo XIX con respecto a las causas del suicidio estaba centrado fundamentalmente en el individuo. De este modo, e independientemente de las fuerzas a las que estuviera sometido o tuviera que gestionar, para el pensamiento de la época el individuo era el responsable último de las decisiones que tomara sobre su vida, y no un mero títere del movimiento social. Sin embargo, como el modelo etiológico se basaba en la presión excesiva que las pasiones ejercían sobre un individuo soberano y dueño de su razón, también se creía que todos aquellos hechos sociales que actuasen debilitando la razón - habitualmente identificada con la moral burguesa - o excitando las pasiones - asociadas comúnmente a la parte más ancestral, peligrosa y vulnerable del ser humano- podían promover el suicidio y las conductas autodestructivas. Por ese motivo, el índice de suicidios fue rápidamente identificado por el discurso higienista como un claro indicador de malestar social, convirtiéndose así, especialmente para los autores de filiación más conservadora, en un tema recurrente de la crítica cultural decimonónica ${ }^{40}$.

39. Así se sugiere en Rosen, George. Orígenes de la psiquiatría social: Tensión social y enfermedad mental desde el siglo XVIII hasta nuestros días. In: Locura y sociedad. Sociología histórica de la enfermedad mental. Madrid: Alianza; 1974, p. 203-227; o Novella, Enric. La ciencia del alma. Locura y modernidad en la cultura española del siglo XIX. Madrid/Frankfurt: Iberoamericana/ Vervuert; 2013, p. 146-147.

40. En Inglaterra apenas se hizo referencia a las cifras de suicidios hasta la década de 1850, probablemente en relación con la falta de datos oficiales (Anderson, Olive. Suicide in Victorian and Edwardian England. Oxford: Clarendon Press; 1987, p. 10). Pero en el resto de Europa, y muy especialmente en Francia, sí hubo continuas alusiones a un aumento alarmante en las cifras de suicidios. Véanse, por ejemplo, Lieberman, n. 7, p. 30-37; Minois, n. 6, p. 315-316; o Martínez Pérez, n. 5, 2001, p. 55-62. 
En el caso español, la religión fue sin duda uno de los elementos predominantes en el discurso médico sobre los trastornos de la voluntad. Dada la profunda influencia que la Iglesia tenía en la vida social, política y científica del país, era comprensible que los debates en torno a un fenómeno moralmente tan controvertido como el suicidio se vieran muy condicionados por sus posiciones, y que los autores católicos franceses tuvieran en este punto una gran circulación e influencia entre los médicos españoles. Así, Brierre de Boismont pensaba que el aumento que él mismo había detectado en las tasas de suicidio estaba muy relacionado con la difusión del individualismo romántico, que había conducido a una exaltación del hedonismo y las pasiones de cuyos estragos daban buena cuenta las trágicas desventuras de René o Werther. A su juicio, la religión era el arma más importante y poderosa para contrarrestar esos extravíos culturales, de manera que las recetas que proponía para contener las pasiones enfermizas y evitar la tendencia al suicidio no eran sino un compendio de la moralidad católica y burguesa: «orden, moderación, paciencia, privaciones, y abstención de los excesos y los vicios» ${ }^{41}$.

Por su parte, el médico y sacerdote trapense Pierre Jean Corneille Debreyne, de quien también se tradujo en España un opúsculo sobre el suicidio, lo consideraba como un acto esencialmente pecaminoso, por lo que entendía que se daba principalmente en los ateos y, en última instancia, como consecuencia del funesto escepticismo y materialismo de la modernidad:

«¿Quiénes son los que se suicidan?, pregunta un periodista cristiano. ¿Es acaso el pobre que va por las calles mendigando el pedazo de pan que sostiene la vida? No, es aquel hombre opulento que acaba de negarle las migajas de su mesa. [...] Una alta civilización sin religión, sin fe, sin costumbres y sin educación religiosa no es más que un azote para los pueblos y hasta para los mismos gobiernos. Miradlo de cerca y podréis seguir los progresos de la civilización en el rastro sangriento de los suicidios» ${ }^{42}$.

\footnotetext{
41. Brierre de Boismont, n. 22, p. 588.

42. Debreyne, Pierre Jean Corneille. Del suicidio considerado bajo los puntos de vista filosófico, religioso, moral y médico, seguido de algunas reflexiones sobre el duelo. Barcelona: Imprenta de Pons y Cia.; 1857, p. 51-70. Es traducción de: Debreyne, Pierre Jean Corneille. Du suicide, considéré aux points de vue philosophique, religieux, moral et médical suivi de quelques réflexions sur le duel. Bruxelles: H. Goemaere; 1854.
} 
Hasta el segundo tercio del siglo XIX, España había sido considerada como un bastión del catolicismo dentro de una Europa crecientemente secularizada. Y los datos sobre el número de suicidios se utilizaban como una señal y amenaza de los daños morales causados por la progresiva pérdida de influencia de las creencias religiosas. Debreyne no dudaba en mencionar a nuestro país como un caso particularmente instructivo:

«El suicidio fue casi desconocido en España mientras el pueblo se mantuvo sinceramente adicto a la religión católica y sobre todo mientras la practicó exactamente. [...] Pues bien, una vez extinguido todo género de fe, acabará tal vez haciéndose general e invencible esa aversión a la vida [...]. Sabido es lo que ha pasado en España desde el año $1834 »{ }^{43}$.

Aquí el autor hacía probablemente referencia a la famosa matanza de frailes del 17 de julio de 1834, motín anticlerical que tuvo lugar en Madrid y se consideró una de las primeras señales del desprestigio de los religiosos en España. Mostraba la hostilidad de parte de las clases populares hacia la Iglesia Católica, ligada al progreso del liberalismo, y causó pánico en la burguesía de la época ${ }^{44}$.

Ciertamente, algunos autores de convicciones liberales se desmarcaron de estas apreciaciones. El barcelonés Rafael Nadal y Lacaba, por ejemplo, describía en un discurso académico pronunciado en 1841 como «una paradoja farisaica» que «el abandono de los principios de nuestra ciencia sublime» pudiera explicar un aumento en la prevalencia del suicidio ${ }^{45}$. Pero, en líneas generales, el énfasis en el valor de la religión para el control de las pasiones y la prevención del «homicidio de sí mismo» se convirtió en un lugar común entre los autores españoles. Así, el jurista Francisco Álvarez Arenas consideraba al ateísmo como una fuente constante de transgresión social, de manera que el suicidio, una de las transgresiones más graves que podían concebirse, no era en el fondo sino un efecto natural de la incredulidad:

\footnotetext{
43. Debreyne, n. 42, p. 23 у р. 73.

44. Sobre dicha revolución se puede consultar: Moliner Prada, Antonio. Anticlericalismo y revolución liberal. In: La Parra, Emilio; Suárez, Manuel. El anticlericalismo español contemporáneo. Madrid: Biblioteca Nueva; 1998.

45. Nadal y Lacaba, Rafael. Suicidios. Barcelona: Imprenta de Antonio Brusi; 1844, p. 11.
} 
«No puede pues extrañar que el hombre que ha adoptado tan fatal doctrina se deje llevar sin resistencia por las pasiones y que, cuando se canse de vivir, eche mano tranquilamente de una pistola o de un puñal» 46 .

Igualmente, tanto Castellví y Pallarés como Pedro Mata subrayaron el papel de contención que la religión ejercía sobre los instintos del sujeto, por lo que también la consideraron como una herramienta útil para evitar la propagación de las conductas autodestructivas ${ }^{47}$. Y, por su parte, siguiendo a Descuret, el mismo Monlau aconsejaba en sus Elementos de higiene pública (1847) la práctica diaria de la oración como «un poderoso muro frente a los ataques continuos de las pasiones», sugiriendo que, antes que cualquier medida coercitiva, la mejor estrategia para la prevención del suicidio no era sino la educación religiosa de la población ${ }^{48}$.

En cualquier caso, y aunque divergieran relativamente en el peso relativo atribuido a la irreligiosidad, una gran mayoría de los médicos españoles coincidían en que los cambios propiciados por la civilización habían supuesto un despertar de las pasiones (individuales y colectivas) y un claro aumento en el número de suicidas. En este sentido, y aparte de las repetidas menciones al materialismo y al hedonismo individualista de los tiempos modernos, muchos autores insistieron en los riesgos derivados del desarrollo de la vida urbana, la ociosidad o incluso el fomento de actividades lúdicas como la lectura o los espectáculos escénicos. Desde su punto de vista, el principal mecanismo de acción por el que dichos factores actuaban sobre el sujeto era la problemática facultad de la imaginación, cuyo estímulo excesivo tendía a excitar desordenadamente las pasiones y podía conducir con facilidad al suicidio ${ }^{49}$. Así, una tesis doctoral presentada en 1862 en la Universidad Central se refería a la imaginación como «el auxiliar de la mayor parte de nuestras pasiones», de manera que «falsea nuestro juicio, sumerge nuestro espíritu en la vaguedad y nos impele a los actos

46. Álvarez Arenas, Francisco. Cuestiones filosófico-político-legales sobre los delitos del suicidio y del duelo. Madrid: Imprenta de la Revista de la Legislación; 1859, p. 39-40.

47. Castellví y Pallarés, n. 27, p. 514; Mata y Fontanet, n. 37, p. 226.

48. Monlau, Pedro Felipe. Elementos de higiene pública. Barcelona: Imprenta de Pablo Riera; 1847, p. 708.

49. Sobre la importancia crítica de la imaginación en el pensamiento médico español del siglo XIX puede verse Novella, Enric J. La higiene del yo: ciencia médica y subjetividad burguesa en la España del siglo XIX. Frenia. 2010; 10: 49-73. 
más desatinados» ${ }^{50}$. Igualmente, el ya citado Nadal y Lacaba consideraba a muchos suicidas víctimas de una imaginación desbocada y mencionaba el ejemplo de algunos literatos, jóvenes cosmopolitas e incluso ciertos sacerdotes, presentando así los efectos nocivos de la imaginación como independientes de la moralidad y el estatus social ${ }^{51}$. Haciendo uso de una terminología más técnica, Maestre de San Juan también señalaba que la civilización era una fuente permanente de excitación cerebral, por lo que destacaba la importancia de abordar el tratamiento del suicida centrándose en la imaginación en tanto función alterada por esta hiperexcitación de origen social:

«La imaginación de los suicidas, al modo de la de los lipemaníacos, concentrada, absorta y dominada por una idea especial que subyuga todas las acciones de su máquina, necesita distracciones que apartando de la imaginación el predominio de ciertas ideas, restablezca el equilibrio perdido» ${ }^{52}$.

Y, por su parte, Monlau recomendaba que el Gobierno prohibiera las obras teatrales y literarias en las que se presentara el suicidio «como un acto sublime y como un recurso soberano en los grandes males», debido justamente al pernicioso ejemplo que ello suponía «para las imaginaciones jóvenes y ardientes, siempre prontas a remedar lo que se les vende bajo apariencias de heroísmo ${ }^{53}$.

Este apunte de Monlau es importante porque, de hecho, la práctica totalidad de los médicos del Ochocientos creían firmemente en la contagiosidad del suicidio en virtud del importante papel de la imitación en la determinación de la conducta de los individuos. Así, se pensaba comúnmente en la imitación como un instinto de gran relevancia para la convivencia, pues permitía adoptar los hábitos y las costumbres impuestas por la sociedad y sancionadas por la tradición. Pero, a pesar de su innegable utilidad, también se alertaba con frecuencia de su potencial peligrosidad, ya que podía conducir igualmente a la difusión de comportamientos socialmente

50. Palomino, Vicente. ¿Cuáles son los caracteres diferenciales entre la monomanía y la pasión? Madrid: Imprenta de Gómez Fuentenebro; 1862, p. 19.

51. Nadal y Lacaba, n. 45, p. 11

52. Maestre de San Juan, n. 35, p. 14.

53. Monlau, n. 48, p. 757. 
inaceptables ${ }^{54}$. Por ese motivo, todas aquellas informaciones periodísticas o manifestaciones literarias o artísticas que presentaran al suicida como un individuo moralmente digno eran consideradas altamente disruptivas por el discurso higienista ${ }^{55}$, por lo que debían ser atajadas con las medidas más enérgicas. Castellví y Pallarés, por ejemplo, llegó a proponer la censura de las obras literarias e incluso de las noticias en la prensa en las que se refirieran casos de suicidio, medida que justificaba alegando haber conocido el caso de una madre de familia que, sin motivo aparente, intentó envenenarse con fósforo tras haber leído en la prensa un caso similar ${ }^{56}$.

Este planteamiento tuvo tal predicamento que siguió siendo muy popular entre los autores de filiación más conservadora hasta finales del siglo XIX. Así, todavía en 1893 La España Moderna publicaba un ensayo del filósofo espiritualista francés Erasmo María Caro en el que se señalaba justamente a la «imitación contagiosa» como una de las causas más comunes de suicidio, particularmente entre niños y adolescentes ${ }^{57}$. Y, en una línea muy similar, el religioso Enrique Gaspar afirmaba en 1896 que el factor causal más importante en el suicida - al que no dudaba en calificar abiertamente como un «faccioso» en virtud de su «soberbio desprecio» por la vida- era «el poder atractivo de la imitación», hasta el punto de equiparar con el cordero las tendencias gregarias del comportamiento humano ${ }^{58}$.

54. Una reflexión sobre el poder de la imitación muy representativa de la época se ofrece en Fabra y Soldevila, Francisco. Filosofía de la legislación natural. Madrid: Imprenta del Colegio de Sordomudos; 1838, p. 35-38. Sobre la importancia de la idea asociada de «contagio moral» para la medicina francesa decimonónica, véase Goldstein, Jan E. «Moral contagion»: A professional ideology of medicine and psychiatry in eighteenth- and nineteenth-century France. In: Geison, Gerald L., ed. Professions and the French State, 1700-1900. Philadelphia: University of Philadelphia Press; 1984, p. 191-222.

55. Citando el testimonio de Madame de Staël, el propio Esquirol afirmaba que el «Werther de Goethe ha producido más suicidios en Alemania que todas las mujeres de este país [...] y lo mismo ha sucedido en Francia después que se ha escrito en favor de él y se ha presentado al público como un acto de nuestro libre albedrío y firmeza». Esquirol, E. [Jean-ÉtienneDominique]. Tratado completo de las enagenaciones mentales. Madrid: Imprenta del Colegio de Sordomudos; 1847, Vol. I, p. 251.

56. Castellví y Pallarés, n. 27, p. 519.

57. Caro, Erasmo María. El suicidio y la civilización. Madrid: La España Moderna; 1893, p. 141.

58. Gaspar, Enrique. El suicidio. Granada: Imprenta de El Pueblo; 1896, p. 7-9. 


\section{Suicidio, degeneración y fin de siglo}

A partir de la década de 1870, la escuela catalana, encabezada por Juan Giné y Partagás, impuso un cambio importante en los modelos teóricos con los que se manejaba la medicina mental española. Alejado del espiritualismo, el modelo de sujeto asumido por este autor estaba más cercano al positivismo y al pensamiento científico-natural, aunque, ciertamente, no renunció a una aproximación psicológica a los trastornos mentales. Así, se ha señalado cómo en algunas partes de su obra, como en la novela de difusión científica Misterios de la locura (1890), Giné define al yo como una instancia modificada por la influencia de las pasiones y que responde a factores psicológicos, describiendo la enfermedad mental desde la subjetividad del que la padece ${ }^{59}$. No obstante, esta noción espiritualista de un yo sustancial y unitario que se encarga de controlar las pasiones se halla ausente en otras obras del autor. En otra novela anterior titulada Un viaje a Cerebrópolis (1884), Giné presenta un modelo de sujeto caracterizado por la articulación fragmentaria de una serie de elementos cognitivos y afectivos condicionados por sus respectivos sustratos orgánicos; la conciencia, identificada aquí con el yo, toma la palabra y se define:

«Señores: No soy lo que parezco, me creen una unidad y soy una suma; [...] yo no soy una unidad sino un total, una suma de entidades que mutuamente se influyen en virtud de orgánicas comunicaciones, que al destruirse provocan irremisiblemente la pérdida de la personalidad psíquica» ${ }^{60}$.

Como puede verse, la voluntad es presentada aquí como el sumatorio de diversas fuerzas de raíz biológica que la alejan definitivamente del concepto tradicional del alma con el que la identificaba la doctrina espiritualista. Y, en consecuencia, el libre albedrío ya no aparece como un dogma resultante de la integridad del yo, sino como una mera ficción:

«La libertad moral es una quimera, una ilusión que aquí todos nos hemos forjado; [...] una sensación genera una idea, esta llama a otra, esta llama a

59. Véase Huertas, Rafael. Memorias de Ultrafrenia: la novela científica y los territorios de la subjetividad. Revista de Estudios Hispánicos. 2010; 44 (1): 31-55.

60. Giné y Partagás, Juan. Un viaje a Cerebrópolis. Barcelona: Imprenta de los Sucesores de Narciso Ramírez; 1884, p. $73-75$ (cursivas en el original). 
otra y así indefinidamente y sin remisión; [...] ¿Es esto libertad o es un fatal automatismo?» ${ }^{61}$.

En su importante Tratado teórico-práctico de frenopatología (1876), Giné ya había identificado pasión e instinto y cuestionado abiertamente la voluntariedad de los actos pasionales: «las conexiones que las regiones pasionales tienen con la sustancia cortical [...] no sabemos si son de carácter voluntario o bien reflejas e inconscientes» ${ }^{62}$. Y, con este planteamiento de fondo, presentaba las conductas suicidas de un modo en el que resulta evidente la influencia del alienista belga Joseph Guislain ${ }^{63}$. Este autor consideraba que la locura era un trastorno de base afectiva y que los distintos cuadros psicopatológicos, muy especialmente aquellos que culminaban en suicidio, partían de un fondo emocional alterado que denominó «frenalgia» o dolor moral ${ }^{64}$. Para Giné, en suma, en todos los casos de suicidio existía igualmente una emoción negativa y esencialmente incontrolable que obraba como causa directa del acto, de manera que la responsabilidad del individuo en la determinación de las conductas autodestructivas era prácticamente nula ${ }^{65}$.

Por su parte, Arturo Galcerán Granés, principal discípulo de Giné, asumió un concepto de voluntad todavía más determinista que el de su maestro. Así, este autor pensaba que el sujeto era dueño de su capacidad volitiva cuando las tensiones psíquicas, expresión de corrientes nerviosas orgánicas, se organizaban de forma articulada, esto es, cuando los estímulos nerviosos de origen inferior eran regulados por asociaciones cerebrales a nivel cortical; si esto no sucedía, actuaban sin freno los centros nerviosos

61. Giné y Partagás, n. 60, p. 79. Sobre este punto puede consultarse Diéguez, Antonio. El problema del libre albedrío en el alienismo español. In: Fuentenebro, Filiberto, Huertas, Rafael; Valiente, Carmen, eds. Historia de la psiquiatría en Europa: Temas y tendencias. Madrid: Frenia; 2003, p. 137-146.

62. Giné y Partagás, Juan. Tratado teórico-práctico de frenopatología. Madrid: Moya y Plaza; 1876, p. 106.

63. Sobre esta influencia de Guislain en Giné y Partagás puede verse Diéguez, Antonio. El problema de la nosografía en la obra psiquiátrica de Giné y Partagás. Asclepio. 1998; 50 (1): 199-221.

64. Guislain señalaba que el humor melancólico se hallaba en el fondo de casi todos los suicidas y definía el suicidio psiquiátrico como un impulso incoercible. Guislain Joseph. Lecciones orales sobre las frenopatías o Tratado teórico y práctico sobre las enfermedades mentales. Madrid: Imprenta de Enrique Teodoro; 1881, Vol. I, p. 187-193. Se trata de la traducción española de: Guislain, Joseph. Leçons orales sur les phrénopathies ou traité theoriqué et pratique des maladies mentales. Gand; L. Hebbelynck; 1852.

65. Giné y Partagás, n. 62, p. 180-181. 
inferiores y aparecía la patología mental ${ }^{66}$. En el caso del suicidio, Galcerán seguía considerando las pasiones como el principal factor causal, aunque las definía de un modo puramente positivista como una corriente nerviosa de origen medular que se ejecutaba en la medida en que generaba un «reflejismo cerebral exagerado»; de este modo, un estímulo emocional de origen subcortical producía una sobrestimulación de la corteza que conducía a la consumación del acto suicida ${ }^{67}$.

En cualquier caso, y a pesar de proponerse interpretaciones cada vez más organicistas de la influencia de las pasiones en la génesis de las conductas autodestructivas, la principal doctrina que inspiró los modelos etiológicos aplicados al suicidio en la España del último cuarto del siglo XIX fue la teoría de la degeneración. Como es sabido, esta doctrina propugnaba un modelo patogénico en el que la enfermedad mental se producía por la herencia enfermiza de los antecesores, a la que se añadía el efecto de diversos factores como el alcoholismo. Y este proceso degenerativo, que se convirtió en el fundamento biológico de la (supuesta) decadencia de la civilización europea del fin de siglo, producía una serie de daños que se reflejaban en la penosa lacra de las enfermedades sociales, siendo el suicidio una de las más destacadas ${ }^{68}$.

Aunque algunos autores como el propio Esquirol o Wilhelm Griesinger habían venido señalando en las décadas previas la influencia del factor here-

66. Galcerán Granés, Arturo. Responsabilidad penal de los enajenados. Datos prácticos para conocerla y graduarla. Gaceta Médica Catalana. 1884; 19: 580-590, 621-629, 648-657, 689-699, 714-722, 745-752.

67. Galcerán Granés, Arturo. Neuropatología y psiquiatría cerebrales. Barcelona: Imprenta de la Casa Provincial de la Caridad; 1895, p. 132-133. El «reflejismo» o simpatía se definía como un fenómeno somático según el cual la disfunción en determinados órganos se manifestaba en otros debido a las conexiones establecidas entre ellos. Sobre este concepto puede verse Plumed, Javier. La etiología de la locura en la psiquiatría española del siglo XIX. Frenia. 2004; IV (2): 69-91 (75-76).

68. Ya el alienista y pionero de la teoría de la degeneración Bénédict-Augustin Morel había considerado el suicidio como un fenómeno estrechamente relacionado con la locura y el delito, poniendo como ejemplo el caso de Suecia. En ese país, a medida que el alcoholismo había aumentado, se habían incrementado la incidencia de casos de suicidio y las tasas de criminalidad, de manera que ambas constituían indicadores objetivos de la extensión social del proceso degenerativo. Véase Morel, Bénédict-Augustin. Traité des dégénérescences de l'espèce humaine. Paris: Baillière; 1857, p. 376-380. Sobre la amplia proyección del degeneracionismo en la España de entresiglos es imprescindible consultar Campos, Ricardo; Martínez Pérez, José; Huertas, Rafael. Los ilegales de la naturaleza. Medicina y degeneracionismo en la España de la Restauración (1876-1923). Madrid: CSIC; 2000. 
ditario en la producción del suicidio, el degeneracionismo lo convirtió en su causa última. En un discurso leído en 1877 en la Facultad de Medicina de la Universidad Central, Matías Zaragoza Aveño señalaba que el verdadero suicidio siempre partía de una predisposición heredada o adquirida, siendo el resto de causas accesorias ${ }^{69}$. Y la misma opinión expresaban unos años después Jesús Sarabia y Pardo, presidente de la Sociedad de Pediatría de Madrid y miembro de la Real Academia de Medicina ${ }^{70}$, y Victoriano Garrido, para quien las causas del suicidio residían en una funesta combinación de «causas naturales como la herencia neuropática»y «causas físico-sociales como el alcoholismo, los traumatismos, las intemperancias de todo género, la índole de la educación, el estado civil y la sociedad» ${ }^{71}$.

Tal como hemos mostrado anteriormente, la imitación era vista como uno de los principales factores que, al modular la influencia del entorno social sobre el individuo, podían contribuir a la aparición de conductas suicidas. Para la doctrina de la degeneración, las causas sociales actuaban de forma directa y biológicamente condicionada sobre la persona y se difundían a través de la herencia, con lo que el individuo se veía impotente para manejar las fuerzas biológicas expresadas en él ${ }^{72}$. En este sentido, Sarabia pensaba que la imitación estaba directamente relacionada con la herencia, ya que

«es preciso que exista, como señala Morel, una anomalía intelectual, que ya manifiesta, lleve a efecto fenómenos que una sana razón rechaza, o ya en estado latente, halle en la imitación la causa determinante de un acto anómalo» ${ }^{73}$.

69. Zaragoza Aveño, Matías. El suicidio en relación con las ciencias médicas. Madrid: s.e.; 1877, p. 7.

70. Sarabia, Jesús. El suicidio como enfermedad social. Madrid: Imprenta de la Sociedad Montera; 1889, 20-21. Sobre este autor, que dedicó buena parte de su obra a cuestiones relacionadas con la higiene y la salud pública, puede verse: Rey, Antonio. Estudios médico-sociales sobre marginados en la España del siglo XIX. Madrid: Ministerio de Sanidad y Consumo, 1990.

71. Garrido, Victoriano. La cárcel o el manicomio. Madrid: Casa Editorial de Don José María Faquineto; s.d. [probablemente 1888], p. 19.

72. Otro autor inspirador del degeneracionismo como Prosper Lucas consideraba que la tendencia a la imitación era un poderoso instinto que facilitaba la transmisión materno-filial de aspectos básicos del psiquismo, como el movimiento y la voz. Y, en consecuencia, postuló un mecanismo de propagación por imitación de enfermedades como las monomanías y las neurosis, así como de conductas patológicas como el alcoholismo (tema al que ya dedicó en 1833 su propia tesis doctoral). Véase Lucas, Prosper. Traité philosophique el physiologique de l'hérédité naturelle dans les états de santé et de maladie du système nerveux. Paris: J.B. Baillière; 1850, Vol. II, p. 559 y 727.

73. Sarabia, n. 70, p. 21. 
Asimismo, Garrido afirmaba que la imitación producía un «contagio moral» en individuos predispuestos, y era un factor causal tanto en casos de suicidio como de homicidio, ya que era capaz de despertar emociones violentas como la cólera y la venganza ${ }^{74}$. Por lo demás, la importancia del factor hereditario era tan dramática que condicionaba no sólo la producción del acto suicida, sino la misma forma en que se ejecutaba. Así, por ejemplo, Joaquín Martínez Valverde afirmaba que la impulsión al suicidio era común en familias con antecedentes de locura y que la forma e incluso el momento en que se consumaba el acto suicida solía ser similar en las distintas generaciones de una misma familia ${ }^{75}$.

Por norma general, los autores degeneracionistas también postulaban un fondo emocional alterado detrás del suicidio. Muchos de estos autores, como Martínez Valverde y Garrido, se vieron influidos por Valentín Magnan, autor fuertemente condicionado por los principios evolucionistas ${ }^{76}$. Así, pensaban que los sentimientos incontrolados que conducían a la acción autolítica implicaban una alteración en la estructura instintiva del ser humano. En este sentido, Martínez Valverde afirmaba que los instintos de conservación del individuo y de la especie se encontraban menoscabados con frecuencia en trastornos impulsivos como el suicidio ${ }^{77}$. Y Garrido, por su parte, tras describir varios casos, los consideraba causados por una liberación del instinto agresivo, una emoción atávica que, si bien —adecuadamente expresada - constituía el motor del desarrollo social, aquí se volvía desadaptativa y doblegaba al instinto de conservación del individuo y la especie ${ }^{78}$. Desde este punto de vista, el individuo degenerado tendía a actuar sin control alguno sobre sus impulsos emocionales, ya que su desequilibrado psiquismo padecía una anomalía hereditaria que le impedía ejercer el libre albedrío sin que su experiencia subjetiva y sus convicciones morales pudieran alterar el curso de los acontecimientos. «El vesánico suicida más

\footnotetext{
74. Garrido, n. 71, p. 151-157.

75. Martínez Valverde, Joaquín. Guía de las enfermedades nerviosas y mentales, con nociones sobre la terapéutica, deontología y medicina legal frenopáticas. Barcelona: José Espasa; 1900, p. 39-40 y p. 212.

76. Sobre la influencia de Magnan sobre la obra de Martínez Valverde puede verse Plumed, Javier. El concepto de manía en la psiquiatría española del siglo XIX. In: Martínez Pérez, José; Estévez, Juan; Del Cura, Mercedes; Blas, Luis Víctor. La gestión de la locura: conocimientos, prácticas y escenarios (España, siglos XIX-XX). Cuenca: Universidad de Castilla La Mancha; 2004, p. 19-63.

77. Martínez Valverde, n. 75, p. 32.

78. Garrido, n. 71, p. 145-146.
} 
fervientemente religioso - sostenía José María Esquerdo en la introducción a la obra citada de Garrido- no suspende el arma por el temor de incurrir en las penas del infierno» ${ }^{79}$. Si el libre albedrío, en suma, no era sino una instancia ilusoria «resultante de múltiples contradictorias o concordantes influencias materiales sobre las que ningún poder espiritual o inmaterial tiene acción correctora ${ }^{80}$, los actos suicidas estaban exentos de cualquier voluntariedad o fallo moral.

En cualquier caso, es importante señalar que el determinismo biológico subyacente a estas afirmaciones no impidió a los autores influenciados por la teoría de la degeneración incluir al suicidio dentro del grupo de las enfermedades sociales, convirtiéndolo en otra señal inequívoca de la corrupción de las costumbres y los conflictos morales que había traído consigo la modernidad. Así, por ejemplo, Jesús Sarabia se refería a las tensiones sociales y a la lucha de clases en sus consecuencias futuras sobre el suicidio: «la crisis indefectible se plantea, la miseria viene a ser el desenlace de tanta ambición y el suicidio el final de $\tan$ grande desequilibrio» ${ }^{81}$. Y por su parte, un jurista como Ambrosio Tapia comentaba las elevadas cifras de suicidio registradas en el año 1897 y se preguntaba si

«¿Habrá influido en ese resultado, tan triste y desconsolador para el progreso moral del país, como una de sus causas generales, el estado de nuestra patria durante el año 1897, que no es para recordar?» ${ }^{82}$.

De hecho, en la literatura médica se hacían repetidas menciones a la propagación de esta enfermedad social en España como consecuencia del cambio de creencias y comportamientos, a la vez que se insistía en la necesidad de establecer medidas higiénicas de carácter público para frenar el progreso del mal. En este sentido, Zaragoza Aveño sugería tanto el estímulo a la publicación de obras morales como la limitación de actividades que, como el juego, consideraba potencialmente inductoras del suicidio. Pero, al igual que sus colegas de mediados de siglo, su recomendación más enfática no era sino «la instrucción en los deberes del cristianismo», pues, en su

\footnotetext{
79. Esquerdo, José María. Prólogo. In: Garrido, n. 71, p. XXI.

80. Garrido, n. 71, p.9.

81. Sarabia, n. 70, p. 25.

82. Tapia, Ambrosio. Los suicidios en Cataluña y, en general, en toda España. Barcelona: Luis Tasso; 1900, p. 233.
} 
opinión, solo él hacía «tener presente al suicida que su vida no es más que un préstamo del Creador y que no puede disponer de ella» ${ }^{83}$.

\section{Conclusiones}

A lo largo del presente trabajo hemos visto cómo el modelo espiritualista de individuo dotado de un yo unitario, capaz de decidir aunque influenciado por las pasiones, tuvo un gran peso en las especulaciones etiológicas en torno al suicidio elaboradas en España hasta la década de 1860 y siguió siendo muy popular entre los sectores médicos más conservadores durante el resto del siglo XIX. El gran peso de la religión en España, unido a la crítica del liberalismo y la modernidad y a la amplia implantación de la doctrina espiritualista como forma de abordar teóricamente al sujeto fueron, indudablemente, factores importantes en este sentido.

Las doctrinas organicistas que empezaron a popularizarse en el país desde la década de 1860 introdujeron un modelo de sujeto fragmentario y condicionado por fuerzas biológicas que cuestionaba el libre albedrío y convertía al suicida en un individuo víctima de tensiones internas que lo desbordaban. Esta tendencia llegó a su apogeo con el degeneracionismo, que consideró el suicidio como una desgraciada consecuencia de instintos naturales mal canalizados y lo convirtió en una grave enfermedad social determinada por la herencia y el propio proceso degenerativo. De este modo, las conductas suicidas - que constituían, como hemos visto, una condición fácilmente subsumible bajo las nuevas categorías de la medicina mental- mantuvieron e incluso reforzaron su importante papel en el contexto de la crítica cultural insistentemente cultivada por los médicos del Ochocientos. Pues, aunque sus causas se entendieran en unos términos más o menos deterministas, siempre podían atribuirse con relativa facilidad a los disruptivos efectos de una crisis civilizatoria que exigía una ilimitada intervención de los médicos en la regulación de los asuntos humanos. 
